\title{
Electrolytes concentration patterns in the three trimesters of pregnancy
}

\author{
A. OMOROGIUWA ${ }^{1 *}$ and M.O. OZOR ${ }^{2}$ \\ ${ }^{I}$ Department of Physiology, School of Basic Medical Sciences, \\ College of Medical Sciences, University of Benin City, Benin City, Nigeria. \\ ${ }^{2}$ Department of Physiology, Faculty of Basic Medical Sciences, \\ College of Medical Sciences, Ambrose Alli University, Ekpoma, Nigeria. \\ *Corresponding author; E-mail: ask4ade2006@yahoo.com ; Tel: +234(0)7039460340
}

\begin{abstract}
The physiologic adaptations of the pregnant woman involve the renal, cardiovascular and other systems of the body. This study aimed at evaluating electrolyte concentrations in the three trimesters of pregnancy. Blood samples were collected by aseptic techniques and the concentrations of electrolytes were determined using standard laboratory methods such as titration for chloride and bicarbonate and flame photometry for sodium and potassium. Eighty healthy volunteers consisting of 20 non-pregnant control and 20 pregnant women in each of the three trimesters of pregnancy participated in the study. The sodium concentrations in $\mathrm{mmol} / \mathrm{L}$ were $135.91 \pm 3.00,136.42 \pm 3.11,135.70 \pm 2.90$, and $136.30 \pm 2.52$, for control, first trimester, second trimester and third trimester subjects respectively. The potassium concentrations in $\mathrm{mmol} / \mathrm{L}$ were $3.51 \pm$ $0.18,3.49 \pm 0.11,3.53 \pm 0.20$, and $3.50 \pm 0.21$, for control, first trimester, second trimester and third trimester subjects respectively. The chloride concentrations in $\mathrm{mmol} / \mathrm{L}$ were $103.50 \pm 3.21,103.60 \pm 2.92,104.70 \pm 3.10$, and 105.0 \pm 3.02 , for control, first trimester, second trimester and third trimester subjects respectively. The Bicarbonate concentrations in $\mathrm{mmol} / \mathrm{L}$ were $25.75 \pm 1.51,26.11 \pm 1.17,25.69 \pm 1.72$, and $26.10 \pm 1.13$, for control, first trimester, second trimester and third trimester subjects respectively. This study revealed that the electrolytes sodium, potassium, chloride and bicarbonate remained unchanged $(p>0.05)$ as pregnancy advanced. This suggests that the kidneys are well adapted to the handling of electrolytes during pregnancy. (c) 2015 International Formulae Group. All rights reserved.
\end{abstract}

Keywords: Sodium, potassium, bicarbonate, chloride, kidney.

\section{INTRODUCTION}

Pregnancy is a sexually transmitted condition of having a developing fetus (Omorogiuwa and Iyawe, 2009). This special state is known to affect the endocrine (Brunton and Russell, 2010), cardiovascular (Dhawan et al., 2005), electrolyte concentration and blood pressure (Obembe and Antai, 2008). The physiologic alterations in these systems may be mistakenly interpreted as abnormal. During normal pregnancy, the renal system has both anatomical and physiologic alterations that are orchestrated by the gravid uterus, increased concentration of progesterone and estrogen. These changes can have attendant effect on plasma volume. For instance, the rise in secretion of estrogen lowers the operating point for osmoregulation of arginine vasopressin and thirst, with an increase in 
plasma volume (Stachenfeld, 2009). However, the increase in plasma volume is not associated with increase in the number of nephrons instead it is a function of the increase in renal vascular and interstitial volume (Strevens et al., 2003). Renal functional capacity is enhanced by marked vasodilatation, increase in renal blood flow with an overall increase in glomerular filtration rate (Obembe et al., 2003). The glomerular filtration rate (GFR) increases by $40-50 \%$ by the end of the first trimester, peaking at $180 \mathrm{~mL} / \mathrm{min}$ and maintained until 36 weeks of gestation. Thus, pregnancy is a volume-expanded state characterized by net sodium retention of 900 to $1000 \mathrm{mEq}$ and 1.1 to $1.6 \mathrm{~L}$ water mediated by a balance between natriuretic and antinatriuretic forces (Cheung and Lafayette, 2013).

The natriuretic, antinatriuretic and hormonal influence of pregnancy may alter the electrolyte concentrations as pregnancy advances. Thus, the results of biochemical tests during pregnancy may therefore differ from the normal reference ranges so they may be mistakenly interpreted as normal. This study is designed to assess the electrolyte concentration patterns in the three trimesters of pregnancy.

\section{MATERIALS AND METHODS Subjects}

A total of 80 women between the ages of 20 and 30 years at Saint Philomena Catholic Hospital volunteered for the study. Sixty of the women were pregnant and were divided into three groups of first, second and third trimester with 20 subjects in each group. The control subjects were non-pregnant staff within the hospital.

\section{Exclusion criteria}

Diabetic, hypertensive and anemic subjects were excluded from the study. Others excluded were subjects with vomiting, diarrhea, chronic alcohol consumption, renal disease and chronic illnesses.

\section{Inclusion criteria}

Booked patients with booking laboratory parameters that are within normal range.

\section{Collection of blood samples}

Blood samples were collected by standard aseptic methods into sample bottles and allowed to clot. Serum was collected for analysis by centrifuging at 2000 revolutions per minute (rmp) for 5 minutes. The serum collected was analyzed for electrolyte concentration.

\section{Determination of serum sodium, potassium, chloride and bicarbonate}

Sodium and potassium ion levels in serum were determined using a flame photometer (Model $410 \mathrm{C}$, Petracourt Ltd, England) at a wavelength of $598 \mathrm{~nm}$ and 769 $\mathrm{nm}$ for sodium and potassium ions respectively (Mencaly et al., 1976). Chloride ion in serum was determined by end point colourimetric titration method (Visweswariah et al., 1972). Serum bicarbonate was estimated by titrimetric method (Mencaly et al., 1976).

\section{Measurement of blood pressure}

Following a rest period of about 30 minutes in the hospital, the systolic and diastolic pressures were measured in each subject on the brachial artery using auscultatory method. Systolic blood pressure was determined by the appearance of the first Korotkoff's sound. Diastolic pressure was determined as the disappearance of the Korotkoff's sound.

\section{Statistical analysis}

Data were presented as mean \pm standard deviation using the Microsoft excel 2010. The student t-test was used for analyses of data and a $\mathrm{p}$ value $<0.05$ was considered statistically significant.

\section{Ethics}

Ethical clearance was obtained from the Ethics and Collaboration Committee of the St Philomena Catholic Hospital where the 
study was done. Informed consent was also obtained from the subjects.

\section{RESULTS}

Eighty subjects took part in the study with twenty subjects in each trimester of pregnancy and twenty control subjects. The mean age in years for the subjects were 25.4 $\pm 2.2,26.2 \pm 2.3, \quad 25.6 \pm 3.0,26.3 \pm 3.8$ for control, first trimester, second trimester and third trimester respectively. The systolic and diastolic blood pressures were 120.1 ${ }^{`} 11.3$ and $77.2 \pm 10.1 \mathrm{mmHg}, 120.4 \pm 9.3$ and 76.5 $\pm 10.0 \mathrm{mmHg}, 119.4 \pm 10.3$ and $78.3 \pm 11.1$ $\mathrm{mmHg}, 121.5 \pm 9.2$ and $79.2 \pm 9.0 \mathrm{mmHg}$ for the control, first trimester, second trimester and third trimester respectively. There was no significant difference $(p>0.05)$ between the systolic and diastolic blood pressures of the test subjects and control.

The table above shows the electrolyte concentrations in the non-pregnant control and the three trimesters of pregnancy. Table 1 indicates that there was no significant difference $(\mathrm{P}>0.05)$ between the electrolyte concentration values for sodium, potassium, chloride and bicarbonate for the control subjects and the test subjects.

\section{DISCUSSION}

Pregnancy is a special state with multisystem adaptive alterations (Omorogiuwa, 2014). The physiologic adaptations of the pregnant woman involve the circulatory, respiratory, digestive, renal, endocrine and metabolic systems (Dhawan et al., 2005; Neeraj et al., 2010). Normal pregnancy is characterized by widespread vasodilation, with increased arterial compliance and decreased systemic vascular resistance. These hemodynamic changes are accompanied by increases in renal perfusion and glomerular filtration rate and sodium excretion (Obembe et al., 2003).

During pregnancy, the significant changes that occur in the various systems are the result of functional and structural adaptations, which are imperative to support alterations in pre-pregnant homeostasis. In this study, the blood pressure which is a function of the cardiovascular system remained unchanged throughout the trimesters of pregnancy. Although there was a decrease in the blood pressure in the second trimester of pregnancy, it was not statistically significant.

Fluid and electrolyte changes also constitute one of the most fundamental changes in normal pregnancy. Fluid retention accounts for between 9 and $10 \mathrm{~kg}$ of the average maternal weight gain (Richard and Emerson, 2002). Water and sodium regulation have been shown to be minimally affected by estradiol or progesterone (or both) administration, suggesting that in young and healthy women, these hormones alter the homeostatic set point around which these

Table 1: The mean electrolyte values for the control and the test subjects in the trimesters of pregnancy.

\begin{tabular}{lcccc}
\hline Parameters & Control & $\begin{array}{c}\text { First } \\
\text { trimester }\end{array}$ & Second trimester & $\begin{array}{c}\text { Third } \\
\text { trimester }\end{array}$ \\
\hline Sodium $(\mathrm{mmol} / \mathrm{L})$ & $135.91 \pm 3.00$ & $136.42 \pm 3.11$ & $135.70 \pm 2.90$ & $136.3 \pm 2.52$ \\
& & $\mathrm{P}>0.05$ & $\mathrm{P}>0.05$ & $\mathrm{P}>0.05$ \\
\hline Potassium $(\mathrm{mmol} / \mathrm{L})$ & $3.51 \pm 0.18$ & $3.49 \pm 0.11$ & $3.53 \pm 0.20$ & $3.50 \pm 0.21$ \\
& & $\mathrm{P}>0.05$ & $\mathrm{P}>0.05$ & $\mathrm{P}>0.05$ \\
\hline Chloride $(\mathrm{mmol} / \mathrm{L})$ & $103.5 \pm 3.21$ & $103.6 \pm 2.92$ & $104.7 \pm 3.10$ & $105.0 \pm 3.02$ \\
& & $\mathrm{P}>0.05$ & $\mathrm{P}>0.05$ & $\mathrm{P}>0.05$ \\
\hline Bicarboanate $(\mathrm{mmol} / \mathrm{L})$ & $25.75 \pm 1.51$ & $26.11 \pm 1.17$ & $25.69 \pm 1.72$ & $26.1 \pm 1.13$ \\
& & $\mathrm{P}>0.05$ & $\mathrm{P}>0.05$ & $\mathrm{P}>0.05$ \\
\hline
\end{tabular}


systems are regulated rather than induce excess fluid or sodium retention or loss (Stachenfeld, 2009). This study revealed that sodium, potassium, chloride and bicarbonate remained unchanged in the three trimesters of pregnancy. This suggest that, the pressure of the gravid uterus on the bladder and the altered neuromuscular function of the striated sphincter (Weidner et al., 2009) which leads to urinary urgency and incontinence (Van Brummen et al., 2006) do not alter the serum concentrations of sodium, potassium, chloride and bicarbonates of pregnant women. However, there are variations in this view; for instance, Akinloye et al. (2013) observed no changes in sodium but significant increase in chloride concentration in first and second trimester compared to non-pregnant controls. They also observed a significant reduction in potassium and bicarbonate in the first and second trimesters of pregnancy. Nevertheless, they opined that these changes were normalized by the third trimester of pregnancy with the values comparable to non-pregnant controls. Studies have shown that the increase in electrolytes as from the third day of conception is accompanied by an increase in renal ionic excretion and that a net retention of sodium, chloride and potassium did not occur until output of these ions is reduced. However sodium and total osmolality were reduced during the last week of pregnancy despite the salt retention, suggesting increased fetal usage Richard and Emerson (2002). Obembe and Antai (2008) reported that pregnancy is expected to waste potassium because pregnant women eat and excrete normal quantities of sodium yet have a high aldosterone and mineralocorticoids. This is contrary to the findings of Beausejour et al. (2003) who reported that pregnant rats cannot handle sodium load.

The reason for the contrary views may be due to the fact that some of the studies were done in rats which have shorter duration of pregnancy with renal functional anatomical alteration that may different from humans. Furthermore, in the studies that involved humans, the parity which is the number of times a woman had given birth to babies beyond the age of viability was not taken into consideration.

\section{Conclusion}

Electrolytes are known to be responsible for the osmolality of the internal environment and their imbalance can be pathological (Coppo, 2001). Our study indicates that the kidneys are well adapted to the handling of electrolyte during pregnancy as there were no difference in electrolyte values between control and the three trimesters of pregnancy. This suggests that there is a homeostatic mechanism regulating the maternal renal changes orchestrated by the hormones of pregnancy, anatomic renal changes and increase fetal usage of electrolytes.

\section{REFERENCES}

Akinloye O, Obikoya OM, Jegede AI, Oparinde DP, Arowojulu AO. 2013. Cortisol plays central role in biochemical changes during pregnancy. Int J Med Biomed Res., 2(1):3-12.

Aslan D, Aslan G, Yamazhan M .2003. Voiding symptoms in pregnancy: an assessment with international prostate symptom score. Gynecol. Obstet. Invest. 6: 55:46.

Beausejour A, Auger K, St. Lous J, Brochu M. 2003. High sodium intake prevents pregnancy induced decrease of blood pressure in rats. Am. J. Physiol. Heart Circ. Physiol., 285(1): H375-H383

Cheung KL, Lafayette RA.2013. Renal Physiology of pregnancy. Adv chronic Kidney Dis., 20(3): 209-214. Doi.10.1053/i.ackd.2013.01.012.

Coppo, JA. 2001. Fistologia Comparadadel Medio Interno. Ed Dunken: Buenos Aires, Argentina; 212-216

Dhawan V Brookes ZLS, Kanfman S. 2005. Repeated Pregnancies (Multiparity) increase venous tone and reduces compliance. Am J. Physiol., 11: 52-59 
Irving SO, Burgess NA. 2002. Managing severe loin pain in pregnancy. BJOG., 109: 1025 .

Mencaly B, Bisits A, Madsen G, McLean M, O'Calaghan S, Smith R, Giles W. 1976. Corticotropin-releasing hormone; biochemical predictor of preterm delivery in a pilot randomized trial of treatment of preterm labour. Am J Obstet Gynecol., 180: 232-241.

Neeraj S, Candy S, John P, Joydeep S, Vaneet K. 2010. Effect of advanced uncomplicated pregnancy on pulmonary function parameters of North Indian Subjects. Indian $J$. Physiol. Pharmacol., 54(1): 69-72.

Nel JT, Diedericks A, Joubert G and Arndt K. 2001. A prospective clinical and urodynamic study of bladder function during and after pregnancy. Int. Urogynecol. J. Pelvic Floor Dysfunct., 12: 21.

Obembe AO, Antai AB, Ibu JO. 2003. Renal function in Pregnant and non-Pregnant Women in Calabar - Nigeria. Niger. J. Health Biomed. Sci., 2(2): 73-77.

Omorogiuwa A. 2014. Pulmonary function and pulse pressure patterns in pregnancy and two days postpartum amongst women in Edo state. Ph.D Thesis, University of Benin, Nigeria, Pp 293.

Omorogiuwa A, Iyawe VI. 2009. Peak Expiratory Flow Rate in the Three
Trimesters of Pregnancy. Journal of Medicine and Biomedical Research, 8(1): 19-23.

Richard KHG, Emerson K. 2002. Physiological response to pregnancy at rest and during exercise. $J$ Appl. Physiol., 36: 529-543.

Stachenfeld NS. 2009. Sex hormones effects on body fluid regulation. Exerc Sports Sci Rev., 36(3): 152-159.

Strevens H, Wide-Swensson D, Hansen A, Horn T, Ingemarsson I, Larsen S. 2003. Glomerular endotheliosis in normal pregnancy and pre-eclampsia. BJOG., 110(9): 831-836.

Van Brummen HJ, Bruinse HW, Van der Bom JG. 2006. How do the prevalences of urogenital symptoms change during pregnancy? Neurourol. Urodyn., 25: 135.

Visweswariah K, Majumder SK, Jayaram MA. 1972. Colorimetric method of estimating hydrolyzable chlorine in lindane (Y-hexachlorocyclohexane) Microchem J., 17: 26-30.

Weidner AC, South MM, Sanders DB, Stinnett SS. 2009. Change in urethral sphincter neuromuscular function during pregnancy persists after delivery. Am. J. Obstet. Gynecol., 201: 529.e1.

Wesnes SL, Rortveit G, Hunskaar S. 2007. Urinary incontinence during pregnancy. Obstet. Gynecol., 109: 922. 